hep-th/0411013

\title{
Open-Closed String Duality from Orbifolded Conifolds
}

\author{
Seungjoon $\mathrm{HyUn}^{a}$ And Kyungho $\mathrm{OH}^{b}$ \\ ${ }^{a}$ Institute of Physics and Applied Physics, \\ Yonsei University, Seoul 120-749, Korea \\ ${ }^{b}$ Department of Mathematics and Department of Physics and Astronomy, \\ University of Missouri-St. Louis, St. Louis, MO 63121, USA
}

\begin{abstract}
We study large $\mathrm{N}$ dualities for a class of $\mathcal{N}=1$ theories realized on type IIB D5 branes wrapping 2-cycles of local Calabi-Yau threefolds which is obtained from resolving orbifolded conifolds or as effective field theories on D4 branes in type IIA brane configurations. The field theory is $\mathcal{N}=1$ supersymmetric $\prod U\left(N_{i j}\right)$ Yang-Mills gauge theory. Strong coupling effects are analyzed in the deformed geometry. We propose open-closed string duality via a geometric transition in toric geometry. The T-dual type IIA picture and M-theory lifting are also considered.
\end{abstract}




\section{Introduction}

Duality between open and closed string is one of the most intriguing features of string theory and impetus for current activities in topological string theory $[1,2,3]$. The large $N$ duality between Chern-Simons theory on the $\mathbf{S}^{\mathbf{3}}$ cycle of the deformed conifold and topological closed strings on the resolved conifold was observed in [4] and the result was embedded in type IIA strings by Vafa [5]. The topological transition becomes a geometric transition between D6 branes on the $\mathbf{S}^{\mathbf{3}}$ of the deformed conifold and type IIA strings on the resolved conifold, with fluxes or between D5 branes wrapped on the $\mathbf{P}^{1}$ cycle of the resolved conifold and type IIB on the deformed conifold with fluxes. The type IIB formulation has been extended to a large class of geometric transition dualities, for various local Calabi-Yau geometries $[6,7,8,9,10,11,12,13,14,15]$.

In the present paper, we begin with orbifolded conifolds $\mathbf{C}_{k l}$ which is an orbifold of the three dimensional conifold $x y-u v=0$ by a discrete group $\mathbf{Z}_{k} \times \mathbf{Z}_{l}$. The resulting singularity is a toric singularity whose equation can be written after coordinate changes as

$$
x y=z^{k}, u v=z^{l}
$$

and we describe a partial resolution of the singularity where the worst singularities are conifolds. Then we take a small resolution of this geometry by replacing each singular point by $\mathbf{P}^{1}$. The normal bundle of $\mathbf{P}^{1}$ will be $\mathcal{O}(-1)+\mathcal{O}(-1)$ and by wrapping $N_{i j}$ D5 branes around each $\mathbf{P}^{1}$ cycles, we obtain $\mathcal{N}=1$ supersymmetric gauge theory with gauge group $\prod_{i=1}^{k} \prod_{j=1}^{l} U\left(N_{i j}\right)$ from type IIB open string theory. Following AdS/CFT, the branes will disappear while fluxes remain through three Lagrangian cycles in a new geometry in the large $N_{i j}$ limit. Now in the strong coupling regime, each gauge group has a gluino condensation. This new geometry is obtained by deforming the partially resolved orbifolded conifold where $\mathbf{P}^{1}$ cycles are shrunken and are replaced by the $\mathbf{S}^{3}$ cycles with RR, NS fluxes. The gluino condensation are mapped into the sizes of $\mathbf{S}^{3}$ cycles which are periods of holomorphic three forms.

The $\mathbf{C}^{*}$ action on the orbifolded conifold by

$$
\lambda \cdot(x, y, u, v, z) \rightarrow\left(\lambda x, \lambda^{-1} y, \lambda u, \lambda^{-1} v, z\right), \lambda \in \mathbf{C}^{*}
$$

on (1.1) naturally extends to the resolved orbifolded conifold and by taking T-dual along the $U(1)$ direction, one obtain type IIA pictures which have been explored in a series of papers $[12,13,16]$. We may now lift this type IIA theory to eleven dimensional M

theory where the brane configuration give rises to a Riemann surface embedded in a complex three dimensional space. 


\section{Vafa's open-closed string dualities}

We will briefly review some of features of Vafa's $\mathcal{N}=1$ open and closed string dualities for large $\mathrm{N}$ which will be used later. Consider type IIB theory on a non-compact CalabiYau threefold $O(-1)+O(-1)$ of $\mathbf{P}^{1}$ which is a small resolution of the conifold :

$$
x y-u v=0
$$

by wrapping $N$ D5 branes on $\mathbf{P}^{1}$. This gives a four dimensional $\mathcal{N}=1 U(N)$ pure Yang-Mills theory described by open strings ending on the D5 branes, in the small 't Hooft parameter regime. Vafa's duality states that in the large $N$ limit (large 't Hooft parameter regime), this is equivalent to type IIB on the deformed conifold:

$$
f=x y-u v-\mu=0 .
$$

In the deformed conifold, the $\mathbf{P}^{1}$ cycle is shrunken and replaced by $\mathbf{S}^{3}$ of size $\mu$ which is identified with the condensate of the $S U(N)$ glueball superfield $S=-\frac{1}{32 \pi^{2}} \operatorname{Tr} W_{\alpha} W^{\alpha}$. The description is now in terms of closed strings. Rather than the $N$ original D 5 branes, there are now $N$ units of RR flux through $\mathbf{S}^{3}$, and also some NS flux through the noncompact cycle dual to $\mathbf{S}^{3}$. The glueball $S$ is identified with the flux of the holomorphic 3 -form on the compact 3-cycle of the deformed conifold

$$
S=\int_{A} \Omega
$$

and the integral of holomorphic 3-form on the noncompact 3-cycle is made with introducing a cut-off $\Lambda_{0}$ :

$$
\Pi=\int_{B}^{\Lambda_{0}} \Omega=\frac{1}{2 \pi i}\left(-3 S \log \Lambda_{0}-S+S \log S\right)
$$

The effective superpotential is written as

$$
W_{\mathrm{eff}}=\int\left(H_{R R}+\tau H_{N S}\right) \wedge \Omega
$$

where $H_{R R}$ is the RR flux on the A-cycle and is due to the $N$ D5 branes and $H_{N S}$ is the NS flux on the noncompact B-cycle. By using the usual IR/UV identification in the AdS/CFT conjecture, we identify the large distance $\Lambda_{0}$ (small IR scale) in supergravity with the small distance (large UV scale) in the field theory such that the coupling constant in field theory is constant and finite in UV. After doing so, the form of the effective superpotential is

$$
W_{\mathrm{eff}}=S \log \left[\Lambda^{3 N} / S^{N}\right]+N S
$$

The condition of supersymmetry implies that the derivative of $W_{\text {eff }}$ with respect to $S$ is zero which implies that $S$ gets $N$ discrete values, separated by a phase. This is the 
gluino condensation in the field theory and signals the breaking of the chiral symmetry $\mathbf{Z}_{\mathbf{2 N}} \rightarrow \mathbf{Z}_{\mathbf{2}}$. The gluons of $S U(N)$ get a mass so the $S U(N)$ gets a mass gap and confines. What remains is the $U(1)$ part of $U(N)$ whose coupling constant is equal to the coupling constant of the $U(N)$ theory divided by $N$.

The lift of the transition to M theory by using MQCD was considered in $[12,13,16]$. A T-duality of the geometrical constructions takes the N D5 branes wrapped on $\mathbf{P}^{1}$ to a brane configuration with two orthogonal NS branes on the directions $x$ and $y$ (together with four directions corresponding to the Minkowski space) and $N$ D4 branes in the the direction $x_{n}$. The lift to M theory involves a single M5 brane which has the worldvolume $R^{1,3} \times \Sigma$ where $\Sigma$ is a 2-dim. manifold holomorphically embedded in $(x, y, t)$ where $t=\exp \left(\frac{x_{n}}{R_{10}}+i x_{10}\right)$. When the $\mathbf{P}^{1}$ cycle shrinks, the direction $x_{n}$ goes to zero and eventually the coordinate $t$ becomes the coordinate of a circle. Because we cannot embed holomorphically into a circle, it results that the coordinate $t$ of the M5 brane become constant and $\Sigma$ is embedded inside $x y=$ const. where the constant is related to the scale of the $U(N)$ theory. After reducing to ten dimensions, $x y=$ const. becomes the equations for a 2-dimensional surface where an NS brane is wrapped, which is T-dual to the deformed conifold and the constant is related to the size of the $\mathbf{S}^{3}$ cycle. Therefore we could explicitly see the relation between the scale of the $U(N)$ theory and the size of the $\mathbf{S}^{3}$ cycle.

The transition has been generalized to more complicated geometries in $[7,8,9,10,11]$, where the blown-up geometry involves more $\mathbf{P}^{1}$ cycles and the deformed geometry involves more $\mathbf{S}^{3}$ cycles. In $[6,11]$, it has been explored that the large $\mathbf{N}$ dualities from type IIB, type IIA and M-theory perspectives for large classes of the $\mathcal{N}=1$ supersymmetric gauge theories by wrapping D5 branes on blown-up $\mathbf{P}^{1}$ cycles in Calabi-Yau threefolds which are obtained by deforming resolved ALE spaces of the ADE singularities. It begins with the $\mathcal{N}=2$ quiver gauge associated with the ADE Dynkin diagrams, which can be geometrically engineered on the resolved ALE spaces. Then the $\mathcal{N}=1$ theory is obtained by adding a superpotential for the adjoint fields, $W_{i}\left(\Phi_{i}\right)$, thus the full tree-level superpotential is

$$
W=\sum_{i=1}^{n} W_{i}-\operatorname{Tr} \sum_{i=1}^{n} \sum_{j=1}^{n} s_{i, j} Q_{i, j} \Phi_{j} Q_{j, i}, \quad \text { where } W_{i}=\operatorname{Tr} \sum_{j=1}^{d_{i}+1} g_{i, j-1} \Phi_{i}^{j},
$$

with adjoints $\Phi$ and bifundamentals $Q_{i, j}$, which fixes the moduli space of the $\mathcal{N}=1$ theory. 


\section{Toric Description of Orbifolded Conifolds}

In this section, we begin with toric description of orbifolded conifolds and the D-brane world-volume gauge theory on its resolution. The orbifolded conifolds have been studied in various aspects $[17,18]$. A toric variety is a space which contains algebraic torus $\left(\mathbf{C}^{*}\right)^{d}$ as an open dense subset. For example, a projective space $\mathbf{P}^{d}=\left(\mathbf{C}^{d+1}-\{0\}\right) / \mathbf{C}^{*}$ is a toric variety because it contains $\left(\mathbf{C}^{*}\right)^{d} \cong\left(\mathbf{C}^{*}\right)^{d+1} / \mathbf{C}^{*} \subset\left(\mathbf{C}^{d+1}-\{0\}\right) / \mathbf{C}^{*}$. As in the case of the projective space, we will express our toric varieties as a quotient space (this can be thought of as a holomorphic quotient in the sense of the Geometric Invariant Theory [19] or as a symplectic reduction as in gauged linear sigma model. In our cases, these two will be the same [20].):

$$
V_{\Delta}=\left(\mathbf{C}^{q}-F_{\Delta}\right) / /\left(\mathbf{C}^{*}\right)^{q-d}
$$

where $q, F_{\Delta}$ and the action of $\left(\mathbf{C}^{*}\right)^{q-d}$ on $C^{q}$ are determined by a combinatorial data $\Delta$. Now we give a description of the combinatorial data $\Delta$ for Gorenstein canonical singularity (i.e. a singularity with a trivial canonical class, $K$ ). Consider vectors $v_{1}, \ldots, v_{q}$ in a lattice $\mathbf{N}=\mathbf{Z}^{d} \subset \mathbf{N}_{\mathbf{R}}=\mathbf{N} \otimes \mathbf{R}=\mathbf{R}^{d}$ in general position. We introduce the corresponding homogeneous coordinates $x_{i}$ for of $\mathbf{C}^{q}-F_{\Delta}$ in the holomorphic quotients. In gauged linear sigma model, these correspond to matter multiplets. There will be $(q-d)$ independent relations

$$
\sum_{i=1}^{q} Q_{i}^{(a)} v_{i}=0, \quad a=1, \ldots, q-d
$$

Here $Q^{(a)}$ 's correspond to the charges of the matter fields under $U(1)^{q-d}$ which is the maximal compact subgroup of $\left(\mathbf{C}^{*}\right)^{q-d}$. The D-term equations will be

$$
\sum_{i=1}^{q} Q_{i}^{(a)}\left|x_{i}\right|^{2}=r_{a}, \quad a=1, \ldots, q-d
$$

In the holomorphic quotient, the charge matrix whose column vectors consist of $Q^{(a)}$ determines the action of $\left(\mathbf{C}^{*}\right)^{q-d}$ on $\mathbf{C}^{q}$ i.e. the action of $\left(\lambda_{1}, \lambda_{2}, \ldots, \lambda_{q-d}\right) \in\left(\mathbf{C}^{*}\right)^{q-d}$ on $\left(x_{1}, \ldots, x_{q}\right) \in \mathbf{C}^{q}$ is given by

$$
\left(\lambda_{1}^{Q_{1}^{(1)}} \lambda_{2}^{Q_{1}^{(2)}} \cdots \lambda_{q-d}^{Q_{1}^{(q-d)}} x_{1}, \lambda_{1}^{Q_{2}^{(1)}} \lambda_{2}^{Q_{2}^{(2)}} \cdots \lambda_{q-d}^{Q_{2}^{(q-d)}} x_{2}, \ldots, \lambda_{1}^{Q_{q}^{(1)}} \lambda_{2}^{Q_{q}^{(2)}} \cdots \lambda_{q-d}^{Q_{q}^{(q-d)}} x_{q}\right)
$$

Here the action can be carried out as written or in two steps, an $\left(\mathbf{R}_{+}\right)^{q-d}$ action and a $U(1)^{q-d}$ action if Kähler. The quotient will depend on the gauge fixing determined by the $\left(\mathbf{R}_{+}\right)^{q-d}$ action i.e. the moment map. In the holomorphic approach, this corresponds to different spaces $F_{\Delta}$ which give rise to (partial) resolutions of the original space $V_{\Delta}$. In toric diagram, this corresponds to different triangulations of a convex cone in $\mathbf{R}^{d}$ 
determined by $\left\{v_{1}, \ldots, v_{q}\right\}$. The collection of these combinatorial data is denoted by $\Delta$ called a fan. The quotient space $V_{\Delta}$ will have Gorenstein canonical singularity if there exists $u \in \mathbf{Z}^{d}$ such that $u \cdot v_{i}=1$ for all $i$ [21]. Thus $v_{i}$ 's will lie on the hyperplane with normal $u$ at a distance $1 /\|u\|$ from the origin in $\mathbf{R}^{d}$. This imposes the following condition on the charge vectors $Q^{(a)}$ :

$$
\sum_{i=1}^{q} Q_{i}^{(a)}=0, \quad a=1, \ldots, q-d
$$

To put our discussions in the language of the gauged linear sigma model, recall that $\mathbf{C}^{q}$ is a symplectic manifold with the standard symplectic form $\omega=i \sum_{i=1}^{q} d z^{i} \wedge d \bar{z}^{\bar{i}}$. The maximal compact subgroup $G:=U(1)^{q-d}$ of $\left(\mathbf{C}^{*}\right)^{q-d}$ acts covariantly on a symplectic manifold $\left(\mathbf{C}^{q}, \omega\right)$ by symplectomorphisms. The infinitesimal action will give rise to a moment map $\mu: \mathbf{C}^{q} \rightarrow \mathbf{g}^{*}$ by Poisson brackets. In coordinates, the components of $\mu: \mathbf{C}^{q} \rightarrow \mathbf{R}^{q-d}$ are given by

$$
\mu_{a}=\sum_{i=1}^{q} Q_{i}^{(a)}\left|x_{i}\right|^{2}-r_{a}
$$

where $r_{a}$ are undetermined additive constants. The symplectic reduction is then defined as

$$
V(r) \equiv \mu^{-1}(0) / G
$$

The structure of $V(r)$ will depend on $r$. It follows from (3.15) that every $\left(\mathbf{C}^{*}\right)^{q-d}$-orbit in $\mathbf{C}^{q}$ will contribute at most one point to $V(r)$. The value of $r$ will determine the contributing orbits. For a fixed $r$, the set of $\left(\mathbf{C}^{*}\right)^{q-d}$-orbits which do not contribute is precisely $F_{\Delta}$. The quotient space $V(r)$ carries a symplectic form $\omega_{r}$ by reducing $\omega$. The symplectic reduction carries a natural complex structure, in which the reduced symplectic form becomes a Kähler form.

Now we will consider quotient singularities of the conifold (i.e. orbifolded conifold). The conifold is a three dimensional hypersurface singularity in $\mathbf{C}^{4}$ defined by:

$$
\mathcal{C}: \quad x y-u v=0 .
$$

The conifold can be realized as a holomorphic quotient of $\mathbf{C}^{4}$ by the $\mathbf{C}^{*}$ action given by $[22,15]$

$$
\left(A_{1}, A_{2}, B_{1}, B_{2}\right) \mapsto\left(\lambda A_{1}, \lambda A_{2}, \lambda^{-1} B_{1}, \lambda^{-1} B_{2}\right) \quad \text { for } \lambda \in \mathbf{C}^{*} .
$$

Thus the charge matrix is the transpose of $Q^{\prime}=(1,1,-1,-1)$ and $\Delta=\sigma$ will be a convex polyhedral cone in $\mathbf{N}_{\mathbf{R}}^{\prime}=\mathbf{R}^{3}$ generated by $v_{1}, v_{2}, v_{3}, v_{4} \in \mathbf{N}^{\prime}=\mathbf{Z}^{3}$ where

$$
v_{1}=(1,0,0), \quad v_{2}=(0,1,0), \quad v_{3}=(0,0,1), \quad v_{4}=(1,1,-1) .
$$


The isomorphism between the conifold $\mathcal{C}$ and the holomorphic quotient is given by

$$
x=A_{1} B_{1}, \quad y=A_{2} B_{2}, \quad u=A_{1} B_{2}, \quad v=A_{2} B_{1} .
$$

We take a further quotient of the conifold $\mathcal{C}$ by a discrete group $\mathbf{Z}_{k} \times \mathbf{Z}_{l}$. Here $\mathbf{Z}_{k}$ acts on $A_{i}, B_{j}$ by

$$
\left(A_{1}, A_{2}, B_{1}, B_{2}\right) \mapsto\left(e^{-2 \pi i / k} A_{1}, A_{2}, e^{2 \pi i / k} B_{1}, B_{2}\right),
$$

and $\mathbf{Z}_{l}$ acts by

$$
\left(A_{1}, A_{2}, B_{1}, B_{2}\right) \mapsto\left(e^{-2 \pi i / l} A_{1}, A_{2}, B_{1}, e^{2 \pi i / l} B_{2}\right) .
$$

Thus they will act on the conifold $\mathcal{C}$ by

$$
(x, y, u, v) \mapsto\left(x, y, e^{-2 \pi i / k} u, e^{2 \pi i / k} v\right)
$$

and

$$
(x, y, u, v) \mapsto\left(e^{-2 \pi i / l} x, e^{2 \pi i / l} y, u, v\right) .
$$

Its quotient is called the hyper-quotient of the conifold or the orbifolded conifold and denoted by $\mathcal{C}_{k l}$. To put the actions (3.20), (3.21) and (3.22) on an equal footing, consider the over-lattice $\mathbf{N}$ :

$$
\mathbf{N}=\mathbf{N}^{\prime}+\frac{1}{k}\left(v_{3}-v_{1}\right)+\frac{1}{l}\left(v_{4}-v_{1}\right)
$$

Now the lattice points $\sigma \cap \mathbf{N}$ of $\sigma$ in $\mathbf{N}$ is generated by $(k+1)(l+1)$ lattice points as a semigroup (These lattice points will be referred as a toric diagram.). The charge matrix $Q$ will be $(k+1)(l+1)$ by $(k+1)(l+1)-3$. The discrete group $\mathbf{Z}_{k} \times \mathbf{Z}_{l} \cong \mathbf{N} / \mathbf{N}^{\prime}$ will act on the conifold $\mathbf{C}^{4} / / U(1)$ and its quotient will be the symplectic reduction $\mathbf{C}^{(k+1)(l+1)} / / U(1)^{(k+1)(l+1)-3}$ with the moment map associated with the charge matrix $Q$. The new toric diagram for $\mathcal{C}_{k l}$ will also lie on the plane at a distance $1 / \sqrt{3}$ from the origin with a normal vector $(1,1,1)$ and we draw a toric diagram on the plane for $\mathcal{C}_{57}$ : The action (3.23), (3.24) of $\mathbf{Z}_{k} \times \mathbf{Z}_{l}$ on the conifold $\mathcal{C}$ can be lifted to an action on $\mathbf{C}^{4}$ whose coordinates are $x, y, u, v$. The ring of invariants will be $\mathbf{C}\left[x^{l}, y^{l}, x y, u^{k}, v^{k}, u v\right]$ and the orbifolded conifold $\mathcal{C}_{k l}$ will be defined by the ideal $(x y-u v) \mathbf{C}\left[x^{l}, y^{l}, x y, u^{k}, v^{k}, u v\right]$. Thus after renaming variables, the defining equation for the orbifolded conifold will be

$$
\mathcal{C}_{k l}: x y=z^{l}, \quad u v=z^{k} .
$$

Figure 1 shows the toric diagram for $\mathcal{C}_{57}$. 


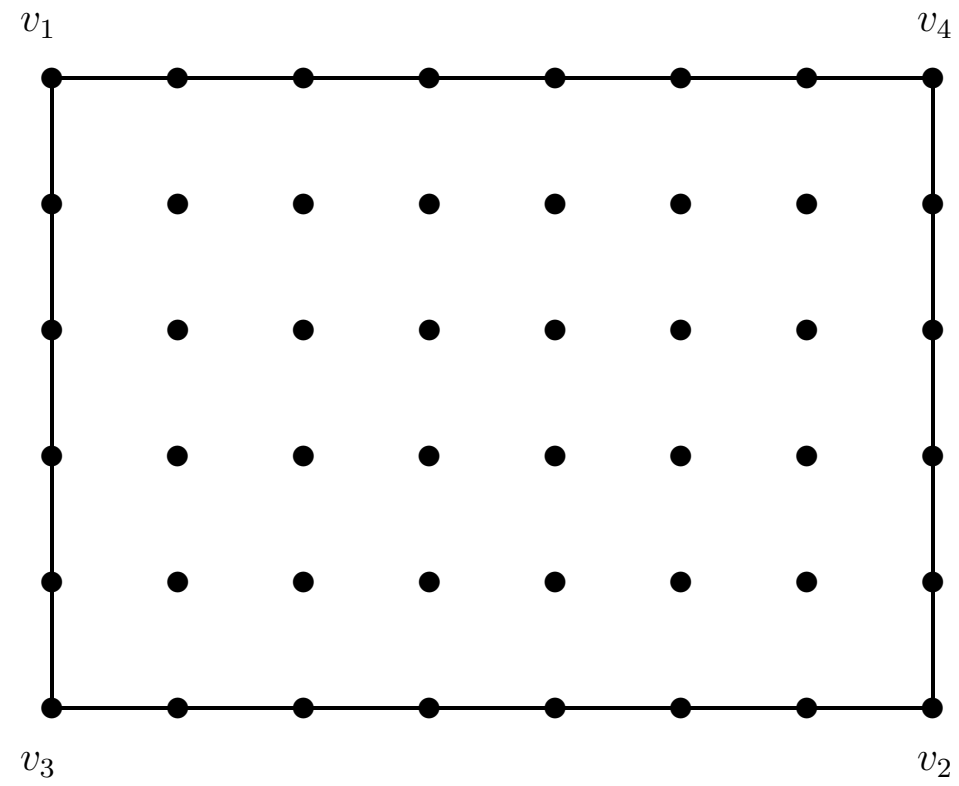

Figure 1: A toric diagram for $\mathbf{Z}_{5} \times \mathbf{Z}_{7}$ hyper-quotient of the conifold, $\mathcal{C}_{57}$

\section{Open String Description on Small resolution of orbifolded conifolds}

We resolve the orbifolded conifold in two steps. First let us consider a partial resolution of the orbifolded conifold where all singularities are conifold singularities. This is obtained by subdivision of the toric diagram. This subdivision is done by adding edges among the adjacent lattice points in the toric diagram i.e. the points in $\sigma \cap \mathbf{N}$. Now there are $k l$ singular points and all of them are conifold singularities. The new toric diagram for this partial resolution for $\mathcal{C}_{57}$ is shown in Figure 2. Now we make a further subdivision of the toric diagram by putting edges between the vertices in the diagonal direction. For each square, we put only one edge and we have two choices i.e. northeast or northwest diagonals. The resulting geometry for this choice are related by flops and they induces Seiberg dualites as explained in $[6,13]$ in the gauge theory we are about to explain. This will create $k l$ new $\mathbf{P}^{1}$ s in the geometry and the local geometry around each $\mathbf{P}^{1}$ 's can be identified with $\mathcal{O}(-1)+\mathcal{O}(-1)$ of $\mathbf{P}^{1}$. The resulting geometry will be called a resolved orbifolded conifold or a small resolution of the orbifolded conifold. By putting $N_{i j}$ D5 branes on each $\mathbf{P}^{1}$, we consider type IIB theory on the world volume of D-branes. This gives a four dimensional $\mathcal{N}=1$ pure Yang-Mills theory described by strings ending on the D5 branes with gauge group

$$
\prod_{i=1}^{k} \prod_{j=1}^{l} U\left(N_{i j}\right) .
$$




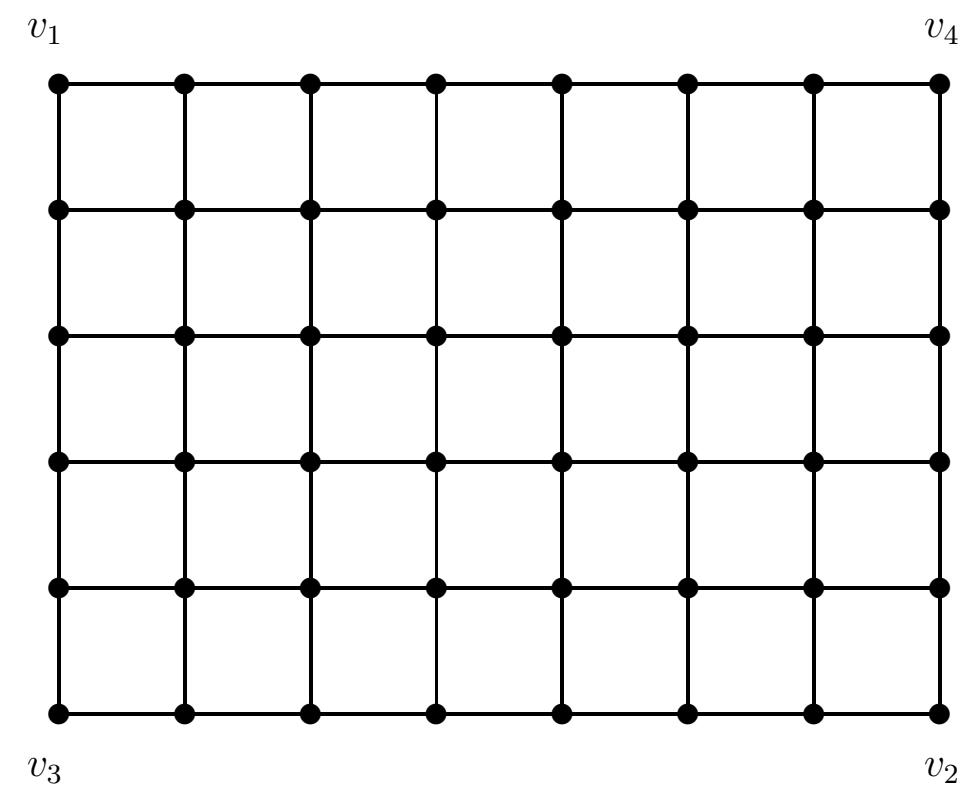

Figure 2: A toric diagram for a partially resolved orbifolded conifold, $\mathcal{C}_{57}$

If the chain of $\mathbf{P}^{1}$ 's where D-branes are wrapped on is disconnected, then there will be no interaction among gauge theories of each connected component in low energy limit. So we will assume that the chain of $\mathbf{P}^{1}$ 's where D-branes are wrapped on is connected. Then there are only two choices for this kind of small resolution. Figure 3 shows one of them for $\mathcal{C}_{57}$ and the other one is obtained by changing the direction of diagonal edges. To relate this to type IIA theory, consider a circle action on the orbifolded conifold

$$
\mathcal{C}_{k l}: x y=z^{l}, \quad u v=z^{k} .
$$

given by

$$
x \rightarrow e^{i \theta} x, y \rightarrow e^{-i \theta} y, u \rightarrow e^{i \theta} u, v \rightarrow e^{-i \theta} v
$$

Then this action lifts to an action on the small resolution of orbifolded conifold and the lifted action acts on the normal bundle $\mathcal{O}(-1)+\mathcal{O}(-1)$ of $\mathbf{P}^{1}$. To describe the action, we introduce two copies of $\mathbf{C}^{3}$ with coordinates $Z, X, Y$ (resp. $Z^{\prime}, X^{\prime}, Y^{\prime}$ ) for the first (resp. second) $\mathbf{C}^{3}$. Then $\mathcal{O}(-1)+\mathcal{O}(-1)$ over $\mathbf{P}^{1}$ is obtained by gluing two copies of $\mathrm{C}^{3}$ with the identification:

$$
Z^{\prime}=\frac{1}{Z}, \quad X^{\prime}=X Z, \quad Y^{\prime}=Y Z
$$

The $Z$ (resp. $Z^{\prime}$ ) is a coordinate of $\mathbf{P}^{1}$ in the first (resp. second) $\mathbf{C}^{3}$ and others are the coordinates of the fiber directions. The blown-down map from the resolved conifold $\mathrm{C}^{3} \cup \mathrm{C}^{3}$ to the conifold $\mathcal{C}$ is given by

$$
x=X=X^{\prime} Z^{\prime}, \quad y=Z Y=Y^{\prime}, \quad u=Z X=X^{\prime}, \quad v=Y=Z^{\prime} Y^{\prime} .
$$




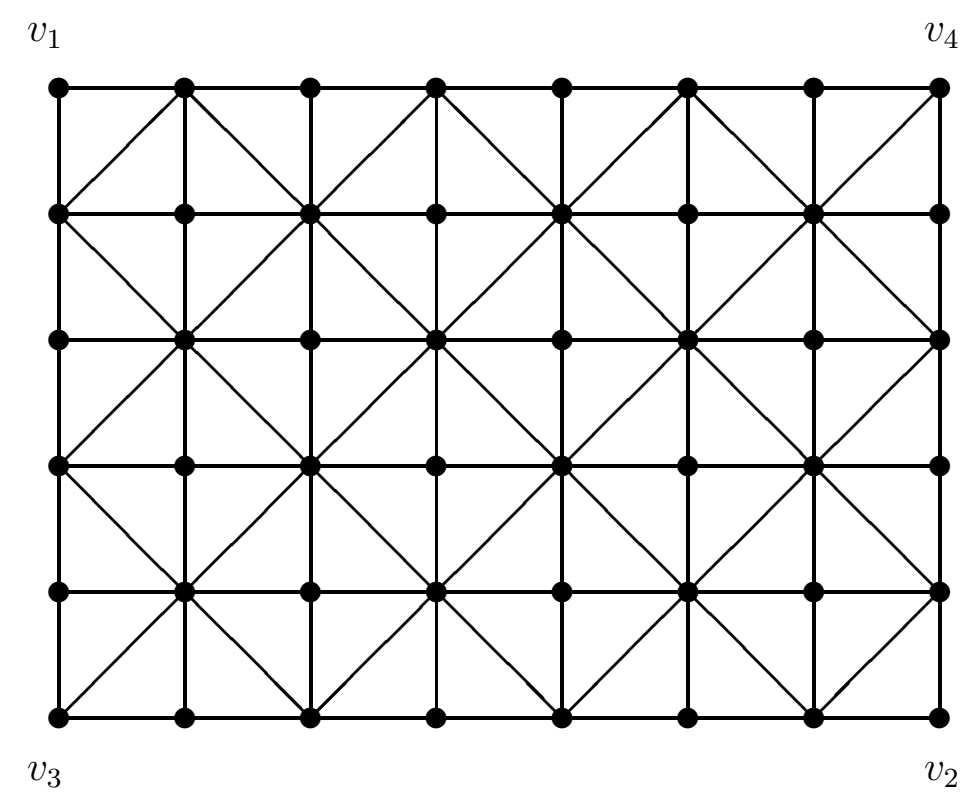

Figure 3: A toric diagram for small resolution of the orbifolded conifold, $\mathcal{C}_{57}$

From this map, one can see that the following action on the resolved conifold is an extension of the action (4.28):

$$
\begin{gathered}
\left(e^{i \theta}, Z\right) \rightarrow e^{i \theta} Z, \quad\left(e^{i \theta}, X\right) \rightarrow X, \quad\left(e^{i \theta}, Y\right) \rightarrow e^{-i \theta} Y \\
\left(e^{i \theta}, Z^{\prime}\right) \rightarrow e^{-i \theta} Z^{\prime}, \quad\left(e^{i \theta}, X^{\prime}\right) \rightarrow e^{i \theta} X^{\prime}, \quad\left(e^{i \theta}, Y^{\prime}\right) \rightarrow Y^{\prime}
\end{gathered}
$$

The orbits degenerates along the union of two complex lines $Z=Y=0$ in the first copy of $\mathbf{C}^{3}$ and $Z^{\prime}=Y^{\prime}=0$ in the second copy of $\mathbf{C}^{3}$. Note that these two lines do not intersect and in fact they are separated by the size of $\mathbf{P}^{1}$. Now we take T-dual along the orbits of $S_{r}$ of type IIB theory obtained by wrapping $N$ D5 branes on the rigid $\mathbf{P}^{1}$. Again there will be two NS branes along the degeneracy loci of the action: one NS brane, denoted by $N S_{X}$, spaced along $X$ direction (which is defined by $Z=Y=0$ in the first $\mathbf{C}^{3}$ ) and the other NS brane, denoted by $N S_{Y^{\prime}}$ along $Y^{\prime}$ direction (which is defined by $Z^{\prime}=X^{\prime}=0$ in the second $\mathbf{C}^{3}$ ). Therefore the T-dual picture will be a brane configuration of D4 brane along the interval with two NS branes in the 'orthogonal' direction at the ends of the the interval. Here the length of the interval is the same as the size of the rigid $\mathbf{P}^{1}$. Hence the type IIA brane picture of the orbifolded conifold will be a web of D4 branes in the interval and NS branes on the intersections. Figure 4 shows the type IIA brane configuration of $\mathcal{C}_{57}$ corresponding to type IIB picture Figure 3 where D4 branes are along the edges and NS branes are located at the dots. 


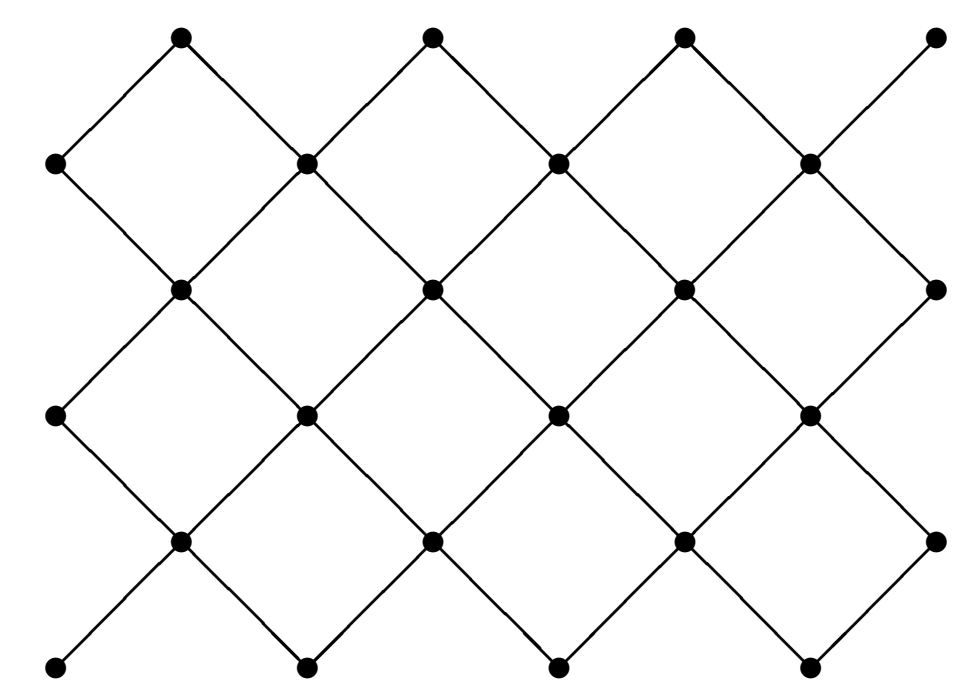

Figure 4: Type IIA brane picture of small resolution of the orbifolded conifold, $\mathcal{C}_{57}$

\section{Closed String Description on Deformed Orbifolded Conifold}

In the large $N_{i j}$ limit in large 't Hooft parameter regime, we have gaugino condensation and mass gap as noticed in [10]. The theory lives on a geometry where the $\mathbf{P}^{1}$ cycles have shrunk and $\mathbf{S}^{3}$ cycles have grown and RR fluxes through them and NS fluxes through their dual cycles have been created. This new geometry is a complex deformation of the partially resolved orbifolded conifold. As one may have noticed, we are only deforming a part of the orbifolded conifold. These are so called normalizable deformations. These normalizable deformations correspond to dynamical fields. The local geometry around $\mathbf{P}^{1}$ cycle where $N_{i j}$ D branes wrapped goes into a geometric transition to a deformed conifold and the deformed geometry is given by an equation

$$
x^{2}+y^{2}+u^{2}+v^{2}=\zeta_{i j}
$$

with holomorphic three form

$$
\Omega \sim \frac{d x d y d u}{\sqrt{\zeta_{i j}-x^{2}-y^{2}-u^{2}}}
$$

Now $\mathbf{P}^{1}$ disappeared and three sphere $A_{i j} \cong \mathbf{S}^{3}$ has been created instead. The three sphere lies as a Lagrangian 3-cycle in the local geometry which is the cotangent bundle 
of $\mathbf{S}^{3}$. The period of the holomorphic three-form $\Omega$ over the 3 -cycle $A_{i j}$ is given by

$$
S_{i j}=\int_{A_{i j}} \Omega \sim \frac{\zeta_{i j}}{4} .
$$

The period over the non-compact dual cycle $B_{i j}$ is divergent and hence we introduce a cut off $\Lambda_{0}$ to regulate the infinity:

$$
\Pi_{i j}=\int_{B_{i j}}^{\Lambda_{0}} \Omega \sim \frac{1}{2 \pi i}\left(-3 S_{i j} \log \Lambda_{0}-S_{i j}+S_{i j} \log S_{i j}\right)+\ldots
$$

As in the other geometric transitions [8,10], $S_{i j}$ is identified with the glueball field $S_{i j}=-\frac{1}{32 \pi^{2}} \operatorname{Tr}_{S U\left(N_{i j}\right)} W_{\alpha} W^{\alpha}$ of the non-Abelian factor $S U\left(N_{i j}\right)$ of $U\left(N_{i j}\right)$ in the dual theory. The $S_{i j}$ will be massive and obtain particular expectation values due to the superpotential $W_{\text {eff. }}$. The dual superpotential $W_{\text {eff }}$ arises from the non-zero fluxes left after the transition. The deformed geometry will have $N_{i j}$ units of $H_{R}$ flux through the $A_{i j} \cong \mathbf{S}^{3}$ cycle due to $N_{i j}$ D5 branes wrapped on the $\mathbf{P}^{1}$ cycle before the transition, and there is also an $H_{N S}$ flux $\alpha_{i j}$ through each of the dual non-compact cycle $B_{i j} \cong \mathbf{R}^{3}$ with $2 \pi i \alpha_{i j}=8 \pi^{2} / g_{0}^{2}$ given in terms of the bare coupling constant $g_{0}$ of the $U\left(N_{i j}\right)$ theory.

Thus the effective superpotential is

$$
\begin{aligned}
-\frac{1}{2 \pi i} W_{\mathrm{eff}} & =\sum_{i=1}^{k} \sum_{j=1}^{l}\left(\int_{A_{i j}} H_{R} \int_{B_{i j}} \Omega-\int_{B_{i j}} H_{N S} \int_{A_{i j}} \Omega\right) \\
& =\sum_{i=1}^{k} \sum_{j=1}^{l}\left(N_{i j} \Pi_{i j}+\alpha_{i j} S_{i j}\right) .
\end{aligned}
$$

After identifying $\Lambda_{0}$ with the UV-cutoff $\Lambda$ in the dual gauge theory, we obtain the usual low energy superpotential associated with the $S U\left(N_{i j}\right)$ glueballs:

$$
W_{\text {eff }}=\sum_{i=1}^{k} \sum_{j=1}^{l} S_{i j}\left(\log \frac{\Lambda^{3 N_{i j}}}{S_{i j}^{N_{i j}}}+N_{i j}\right) .
$$

Integrating out the massive $S_{i j}$ by solving

$$
\frac{\partial W_{\text {eff }}}{\partial S_{i j}}=0
$$

leads to $N_{i j}$ supersymmetric vacua of $S U\left(N_{i j}\right) \mathcal{N}=1$ supersymmetric Yang-Mills:

$$
\left\langle S_{i j}\right\rangle=\exp \left(2 \pi i m / N_{i j}\right) \Lambda^{3}, \quad m=1, \ldots, N_{i j} .
$$

The dual theory obtained after the transition is an $\mathcal{N}=2$

$$
\prod_{i=1}^{k} \prod_{j=1}^{l} U(1) \equiv U(1)^{k l}
$$


gauge theory broken to $\mathcal{N}=1 U(1)^{k l}$ by the superpotential $W_{\text {eff }}$ (5.36) [23]. The $S_{i j}$, which are the same $\mathcal{N}=2$ multiplet as the the $U(1)^{k l}$, get masses and frozen to

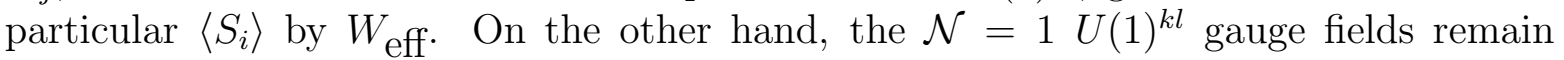
massless. The couplings $\tau_{i j i^{\prime} j^{\prime}}$ of these $U(1)^{\prime}$ 's can be determined by $\Pi_{i j}$ or the $\mathcal{N}=2$ prepotential $\mathcal{F}\left(S_{i j}\right)$, with $\Pi_{i j}=\partial \mathcal{F} / \partial S_{i j}$ :

$$
\tau_{i j i^{\prime} j^{\prime}}=\frac{\partial \prod_{i j}}{\partial S_{i^{\prime} j^{\prime}}}=\frac{\partial^{2} \mathcal{F}\left(S_{i j}\right)}{\partial S_{i j} \partial S_{i^{\prime} j^{\prime}}} .
$$

The couplings should be evaluated at the vacua $\left\langle S_{i j}\right\rangle$ obtained in (5.39). It is possible to consider $\mathrm{M}$ thoery by lifting type IIA brane configuration which is constructed in the previous section and investigate the large N limit via Witten's MQCD formalism. We denote the finite direction of D4 branes by $x^{7}$ and the angular coordinate of the circle $\mathbf{S}^{1}$ in the 11-th dimension by $x^{10}$. Thus the NS branes are separated along the $x^{7}$ direction. We combine them into a complex coordinate

$$
t=\exp \left(-R^{-1} x^{7}-i x^{10}\right)
$$

where $R$ is the radius of the circle $\mathbf{S}^{1}$ in the 11-th dimension. In MQCD [24], the classical type IIA brane configuration turns into a single fivebrane whose world-volume is a product of the Minkowski space $\mathbf{R}^{1,3}$ and the M-theory curve $\Sigma$ in a flat Calabi-Yau manifold

$$
M=\mathbf{C}^{2} \times \mathbf{C}^{*},
$$

whose coordinates are $u, v, t$. Classically, the $u, v$-coordinates of the M-theory curve $\Sigma$ describes the location of NS branes in the type IIA picture. By lifting this to the M-theory, the location of the D4 branes on the NS brane will be smeared out as D4 brane acquire 11-th direction. So, at quantum level, the M-theory curve is obtained by merging tubular neighborhood around edges in Figure 4 which represents D4 branes and NS branes located dots in Figure 4. A lifting from the compact part of the Jacobian of the curves to the non-compact part can be viewed as a geometric transition from M-theory point of view [12].

\section{Acknowledgments}

We would like to thank Jae-Suk Park, Jong-Dae Park and Sang-Heon Yi for inspiring discussions and encouragement. This work was supported by Brain Pool program No. 031S-1-6 from The Korean Federation of Science and Technology Societies. K.O. thanks IPAP at Yonsei University for warm hospitality during his visit. 


\section{References}

[1] A. Iqbal, N. Nekrasov, A. Okounkov and C. Vafa, "Quantum foam and topological strings," arXiv:hep-th/0312022.

[2] M. Aganagic, R. Dijkgraaf, A. Klemm, M. Marino and C. Vafa, "Topological strings and integrable hierarchies," arXiv:hep-th/0312085.

[3] H. Ooguri, A. Strominger and C. Vafa, "Black hole attractors and the topological string," arXiv:hep-th/0405146.

[4] R. Gopakumar and C. Vafa, "On the gauge theory/geometry correspondence," Adv. Theor. Math. Phys. 3 (1999) 1415 [hep-th/9811131].

[5] C. Vafa, "Superstrings and topological strings at large N," J. Math. Phys. 42, 2798 (2001) [hep-th/0008142].

[6] K. Oh and R. Tatar, "Duality and confinement in $\mathrm{N}=1$ supersymmetric theories from geometric transitions," Adv. Theor. Math. Phys. 6, 141 (2003) [hepth/0112040].

[7] S. Sinha and C. Vafa, "SO and Sp Chern-Simons at large N," arXiv:hep-th/0012136.

[8] F. Cachazo, K. A. Intriligator and C. Vafa, "A large N duality via a geometric transition," Nucl. Phys. B 603, 3 (2001) [hep-th/0103067].

[9] J. D. Edelstein, K. Oh and R. Tatar, "Orientifold, geometric transition and large N duality for SO/Sp gauge theories," JHEP 0105, 009 (2001) [hep-th/0104037].

[10] F. Cachazo, S. Katz and C. Vafa, "Geometric transitions and N = 1 quiver theories," arXiv:hep-th/0108120.

[11] F. Cachazo, B. Fiol, K. A. Intriligator, S. Katz and C. Vafa, "A geometric unification of dualities," Nucl. Phys. B 628 (2002) 3 [hep-th/0110028].

[12] K. Dasgupta, K. Oh, R. Tatar, "Geometric Transition, Large N Dualities and MQCD Dynamics", Nucl. Phys. B 610 (2001) 331 [hep-th/0105066].

[13] K. Dasgupta, K. Oh and R. Tatar, "Open/closed string dualities and Seiberg duality from geometric transitions in M-theory, JHEP 0208 (2002) 026 [hep-th/0106040].

[14] I. R. Klebanov and M. J. Strassler, "Supergravity and a confining gauge theory: Duality cascades and chiSB-resolution of naked singularities," JHEP 0008 (2000) 052 [arXiv:hep-th/0007191], I. R. Klebanov and A. A. Tseytlin, "Gravity duals of supersymmetric SU(N) x SU(N+M) gauge theories," Nucl. Phys. B 578 (2000) 123 
[arXiv:hep-th/0002159], I. R. Klebanov and N. A. Nekrasov, "Gravity duals of fractional branes and logarithmic RG flow," Nucl. Phys. B 574 (2000) 263 [arXiv:hepth/9911096].

[15] I. R. Klebanov and E. Witten, "Superconformal field theory on threebranes at a Calabi-Yau singularity," Nucl. Phys. B 536 (1998) 199 [arXiv:hep-th/9807080].

[16] K. Dasgupta, K. Oh, J. Park and R. Tatar, "Geometric transition versus cascading solution", JHEP 0201 (2002) 031 [hep-th/0110050].

[17] K. Dasgupta, S. Hyun, K. Oh and R. Tatar, "Conifolds with discrete torsion and noncommutativity," JHEP 0009, 043 (2000) [arXiv:hep-th/0008091].

[18] K. Oh and R. Tatar, "Branes at orbifolded conifold singularities and supersymmetric gauge field theories," JHEP 9910, 031 (1999) [arXiv:hep-th/9906012].

[19] D. Mumford, J. Forgarty, F. Kirwan, Geometric Invariant Theory, (Springer 1992)

[20] F. C. Kirwan, Cohomology of quotients in symplectic and algebraic geometry, (Princeton University Press, 1984)

[21] M. Reid, "Minimal Models of Canonical 3-folds", pp.131-180, Advanced Stuides in Pure Mathematics 1, ed. S. Iitaka, Kinokuniya (1983)

[22] E. Witten, "Phases of N=2 Theories in Two Dimensions", Nucl. Phys. B403 (1993) 159 [hep-th/9301042].

[23] T. R. Taylor and C. Vafa, "RR flux on Calabi-Yau and partial supersymmetry breaking," Phys. Lett. B 474, 130 (2000) [hep-th/9912152], P. Mayr, "On supersymmetry breaking in string theory and its realization in brane worlds," Nucl. Phys. B 593, 99 (2001) [hep-th/0003198], G. Curio, A. Klemm, D. Lust and S. Theisen, "On the vacuum structure of type II string compactifications on Calabi-Yau spaces with H-fluxes," Nucl. Phys. B 609, 3 (2001) [hep-th/0012213], G. Dall'Agata, "Type IIB supergravity compactified on a Calabi-Yau manifold with H-fluxes," JHEP 0111, 005 (2001) [hep-th/0107264],L. Andrianopoli, R. D'Auria and S. Ferrara, "Supersymmetry reduction of N-extended supergravities in four dimensions," [hepth/0110277]

[24] E. Witten, "Branes and the dynamics of QCD," Nucl. Phys. Proc. Suppl. 68 (1998) 216. 